\title{
POSITIVE DEFINITE MEASURES WITH APPLICATIONS TO A VOLTERRA EQUATION
}

BY

\author{
OLOF J. STAFFANS
}

ABSTRACT. We study the asymptotic behavior of the solutions of the nonlinear Volterra integrodifferential equation

$$
x^{\prime}(t)+\int_{0}^{t} g(x(t-\tau)) d \mu(\tau)=f(t),
$$

with a positive definite kernel $\mu$. In particular, we give new sufficient conditions on the kernel $\mu$, which together with standard assumptions on $f$ and $g$ yield results on boundedness and asymptotic behavior of the solutions of (*). Our proofs are based on the theory of distribution Fourier transforms.

Introduction. A real nonlinear Volterra integrodifferential equation with a convolution kernel is an equation of the form

$$
x^{\prime}(t)+\int_{0}^{t} a(t-\tau) g(x(\tau)) d \tau=f(t) \quad(0 \leqslant t<\infty) ; \quad x(0)=x_{0},
$$

where $a, g$ and $f$ are given real-valued functions, $x_{0}$ is a given real constant, and $x$ is the unknown solution (prime denotes differentiation). We are basically interested in asymptotic properties of a solution of (1) such as the boundedness on $[0, \infty)$ of a solution, and the question of whether a solution $x$ satisfies

$$
\lim _{t \rightarrow \infty} g(x(t))=0
$$

(with the appropriate assumptions on $a, f$ and $g$ the condition (2) will imply $\lim _{t \rightarrow \infty} x^{\prime}(t)=0$, and the existence of $\left.\lim _{t \rightarrow \infty} x(t)\right)$.

In the existing literature on this nonlinear problem the hypotheses on $g$ and $f$ have, in general, included $g \in C(-\infty, \infty), f \in L^{1}(0, \infty)$, and we shall assume throughout that these two conditions are satisfied (however, in some recent papers the condition $f \in L^{1}(0, \infty)$ has been relaxed to $\lim _{t \rightarrow \infty} f(t)=0$, but then stronger conditions are needed on $a$ and $g$, see [10, Theorem 2.5], [13, Theorem $8 \mathrm{a}]$ and [15, Theorem 3]; sometimes also certain perturbation terms of bounded variation have been allowed, see $[6$, Theorem 1$],[12$, Theorem 1] and $[16$,

Received by the editors March 20, 1975.

AMS (MOS) subject classifications (1970). Primary 45D05, 45G99, 45J05, 45M05; Secondary 42A68, 42A88, 44A10, 46F10.

Key words and phrases. Volterra equation, nonlinear integrodifferential equation, asymptotic stability, positive definiteness, strict positive definiteness, Fourier transform, distributions, weak* convergence. 
Theorem (5.7)]). The first studies on the asymptotic properties of (1) were restricted to the special case of a nonnegative, nonincreasing and convex kernel $a$, and for some time this was the case most intensively investigated, see e.g. [6], [9], [11] , [12] and [14]. The earliest attempt to generalize some of these results to positive definite kernels (in the sense of Bochner, or even more generally, in the sense of Cooper [2]) was made by Halanay [4], using a Popov type technique. A further development of his approach has resulted in a unified theory which covers most of the known results for convex kernels (here we restrict our attention to theorems which permit truly nonlinear functions $g$, and not only small perturbations of linear functions). The following paragraph contains a very brief discussion of this positive definite case, and for more details we refer the reader to [18] and to $\S 6$ below.

One can prove boundedness of the solutions of (1) using weaker assumptions than are needed to prove (2) (this is not necessarily true if one relaxes our requirement $\left.f \in L^{1}(0, \infty)\right)$. Positive definiteness of the kernel $a$ together with some sign and growth conditions on $g$ at infinity implies boundedness of the solutions of (1), and an additional positivity condition on the kernel (together with a tauberian condition) then implies (2). This additional condition on the kernel, originally discovered by Halanay [4], and later named strong positive definiteness by MacCamy and Wong [16], demands that one be able to subtract an exponential from the kernel and still have something which is positive definite. Most nonnegative, nonincreasing and convex kernels are strongly positive definite; however, there exist some that are not strongly positive definite, but for which one can show that a solution $x$ of (1) satisfies (2), see [6, Theorem 1(ii)] and [18, $\S 4]$. In [21] we introduced a new concept, strict positive definiteness, which is weaker than strong positive definiteness, but which still implies (2). The main purpose of this study is to show that strict positive definiteness of the kernel $a$ is equivalent to strict positivity of the real part of the Fourier transform of $a$. This is analogous to the fact that positive definiteness of the kernel $a$ is equivalent to nonnegativity of the real part of the Fourier transform of $a$ (see $\S 1$ below). In particular, all nonnegative, nonincreasing and convex kernels, for which the solutions of (1) are known to satisfy (2), are strictly positive definite.

We have based our treatment below on the concept of positive definite, locally finite Borel measures rather than on the concept of positive definite, locally integrable functions. This greater generality, which is quite desirable for the applications to Volterra equations, causes no major additional difficulties. We first study the properties of positive definite measures (Definitions 1.2 and 1.3 below) and their Fourier transforms in $\S 1$. After that we investigate strictly positive definite measures (Definition 2.1) in $\$ \S 2-3$. To facilitate a comparison with earlier results, in particular those concerning strong positive definiteness, we 
study the Laplace transforms of positive and strictly positive definite measures in $\S \S 4-5$. Finally, in $\S 6$ we give two theorems on the solutions of a slightly generalized version of (1), concerning the existence and boundedness of solutions and the validity of (2), together with some supplements on the uniform continuity of the solutions on $[0, \infty)$.

We shall throughout use the following function, measure or distribution spaces, defined on an interval $(a, b)$ (this open interval is used only for illustration, and can sometimes be replaced by a half-open or closed interval; the specific interval $(-\infty, \infty)$ will frequently be left out from the notation):

$C(a, b)$ : continuous functions,

$C^{\infty}(a, b)$ : infinitely differentiable functions,

$B V(a, b)$ : functions of bounded variation,

$L^{1}(a, b)$ : Lebesgue integrable functions,

$L^{2}(a, b)$ : Lebesgue square integrable functions,

$L^{\infty}(a, b)$ : essentially bounded, measurable functions,

$M(a, b)$ : see Definition 1.1 and Remark 1.1,

$P(a, b)$ : see Definition 1.1 and Remark 1.1,

$S P(a, b)$ : see Definition 2.2,

$P D(a, b)$ : see Definitions 1.2 and 1.3,

$\operatorname{SPD}(a, b)$ : see Definition 2.1,

$O(a, b): C^{\infty}$ functions with compact support in $(a, b)$,

$D^{\prime}(a, b)$ : distributions [22, p. 24],

$S:$ rapidly decreasing testfunctions [22, pp. 233-234],

$S^{\prime}:$ tempered distributions [22, p. 237],

$B^{\prime}$ : bounded distributions [22, p. 200].

We shall also use the following notations ( $\varphi$ stands for an element of some function space, and $u$ for an element of the corresponding dual space, e.g. $\varphi \in D$ and $\left.u \in D^{\prime}\right):$

$\langle\varphi, u\rangle: u$ evaluated at $\varphi$,

$\bar{\varphi}$ : complex conjugate of $\varphi$,

$\bar{u}:\langle\varphi, \bar{u}\rangle=\langle\overline{\bar{\varphi}}, u\rangle$,

$\hat{\varphi}: \hat{\varphi}(\omega)=\int_{-\infty}^{\infty} \varphi(t) e^{-i \omega t} d t(-\infty<\omega<\infty)$,

$\hat{u}:\langle\varphi, \hat{u}\rangle=\langle\hat{\varphi}, u\rangle$,

$\tilde{\varphi}: \tilde{\varphi}(t)=\bar{\varphi}(-t)$,

$\tilde{u}:\langle\varphi, \tilde{u}\rangle=\overline{\langle\widetilde{\varphi}, u}\rangle$,

$\Pi=\{s \in \mathrm{Cl} \operatorname{Re} s>0\}$,

$u^{*}: u^{*}(s)=\left\langle e_{s}, u\right\rangle(s \in \Pi)$, where $e_{s}(t)=e^{-s t}(-\infty<t<\infty)$,

$f * g:(f * g)(t)=\int_{-\infty}^{\infty} f(t-\tau) g(\tau) d \tau$ (see also [20, Definition 7.18]),

$f \circ g:(f \circ g)(t)=f(g(t))$,

$|\mu|$ : the total variation measure of $\mu$. 
With the exception of $\$ 6$ all our functions, measures and distributions are complex-valued, unless explicitly stated otherwise. We identify a measure $\mu$ (or a function) with a distribution by the formula $\langle\varphi, \mu\rangle=\int_{-\infty}^{\infty} \varphi(t) d \mu(t)(\varphi \in D)$. The word "positive" is used as a synonym to "nonnegative", and we write "strictly positive" whenever we want to exclude zero. We extend all functions and measures, defined on some interval $I$, to $(-\infty, \infty)$ by zero outside $I$, unless we give a specific statement to the contrary (so e.g., $D(0, \infty) \subset D(-\infty, \infty)$, but $C[0, \infty) \not C(-\infty, \infty))$.

1. Positive definite measures. In this section we give the basic results on positive definite measures and their Fourier transforms. These measures form a subclass of Schwartz's positive definite distributions, which contains all positive definite functions in the sense of Cooper [2]. The proofs are based on [22].

DEFINITION 1.1. By a locally finite (Radon) measure $\mu$ on $(-\infty, \infty)(\mu \in$ $M(-\infty, \infty)$ ) we mean a complex-valued set function that restricted to subsets of compact sets of $(-\infty, \infty)$ is a complex Borel measure. By $M[0, \infty)$ ("locally finite measures on $[0, \infty)$ ") we mean the subclass of $M(-\infty, \infty)$ vanishing on $(-\infty$, $0)$. The class of positive, locally finite, Borel measures on $(-\infty, \infty)$ is denoted by $P(-\infty, \infty)$.

REMARK 1.1. One can identify $M(-\infty, \infty)$ with the class of distributions of order zero $[22$, p. 25$], M[0, \infty)$ with the class of distributions of order zero, vanishing on $(-\infty, 0)$, and $P(-\infty, \infty)$ with the class of positive distributions [22, p. 29]. We shall make frequent use of these identifications without any further comments.

Definition 1.2. A measure $\mu \in M[0, \infty)$ is positive definite $(\mu \in P D[0, \infty))$ if for every $T>0$ and for every $\varphi \in C[0, T]$,

$$
\operatorname{Re}\left\{\int_{[0, T]} \bar{\varphi}(t) \int_{[0, t]} \varphi(t-\tau) d \mu(\tau) d t\right\} \geqslant 0 .
$$

Definition 1.2 is a straightforward generalization to measures of the class of positive definite functions used in [4], [16], [18] and [21]. A seemingly slightly different class of positive definite functions, defined on $(-\infty, \infty)$ instead of $[0, \infty)$, has also been studied, e.g. by Cooper [2]. His definition generalizes to measures as follows:

DEFINITION 1.3. A measure $\nu \in M(-\infty, \infty)$ is positive definite $(\nu \in$ $P D(-\infty, \infty))$ if for every $T>0$ and for every $\varphi \in L^{2}(-T, T)$,

$$
\int_{[-T, T]} \bar{\varphi}(t) \int_{[t-T, t+T]} \varphi(t-\tau) d \nu(\tau) d t \geqslant 0 .
$$

Note that the double integral in (1.2) is required to be real, and that a larger class of functions $\varphi$ is admitted.

REMARK 1.2. The easiest way to avoid measurability problems in (1.2) is to replace an arbitrary, Lebesgue measurable $\varphi$ by a Borel measurable $\varphi_{1}$, which 
is equal to $\varphi$ a.e. $[m]$ ( $m$ denotes Lebesgue measure). The measure $\nu$ restricted to $[-2 T, 2 T]$ is finite, and one can then use Fubini's theorem to show that the integral in (1.2) is well defined, i.e. absolutely convergent and independent of $\varphi_{1}$ as long as $\varphi_{1}=\varphi$ a.e. $[\mathrm{m}]$.

REMARK 1.3. Extending $\varphi$ to $(-\infty, \infty)$ to be zero outside $[-T, T]$ and using Fubini's theorem one can write (1.2) in the form

$$
\langle\overline{\varphi * \widetilde{\varphi}}, \nu\rangle \geqslant 0 \text {. }
$$

The classes $P D[0, \infty)$ and $P D(-\infty, \infty)$ are closely related to each other; in fact they are essentially equivalent in a certain sense:

Theorem 1.1. Let $\mu \in P D[0, \infty)$. Define $\nu \in M(-\infty, \infty)$ by

$$
\nu=\mu+\tilde{\mu} \text {. }
$$

Then $\nu \in P D(-\infty, \infty)$. Conversely, let $\nu \in P D(-\infty, \infty)$. Take any real number $\alpha$ and define $\mu \in M[0, \infty)$ by

$$
\mu(E)=(1 / 2+\alpha i) \nu(E \cap\{0\})+\nu(E \cap(0, \infty))
$$

for every bounded Borel set $E \subset(-\infty, \infty)$. Then $\mu \in P D[0, \infty)$. Moreover, every $\nu \in P D(-\infty, \infty)$ satisfies $\nu=\widetilde{\nu}$ (in particular, $\nu(\{0\})$ is real), and hence the restriction (1.5) followed by the extension (1.4) reproduces the original measure $\nu$.

REMARK 1.4. With the exception of the point $\{0\}$, formula (1.5) simply defines the restriction of $v$ to $[0, \infty)$, whereas (1.4), again with the exception of the point $\{0\}$, defines $\nu$ to be the extension to $(-\infty, \infty)$ of $\mu$ satisfying $\nu=\widetilde{\nu}$. Formulas (1.4) and (1.5) are equivalent provided $\nu=\widetilde{\nu}$ and $\alpha=\operatorname{Im}\{\mu(\{0\})\}$.

The proof of Theorem 1.1 is based on the following lemma.

Lemma 1.1. Let $\mu \in M[0, \infty)$, and define $\nu$ by (1.4). Then for every $T>$ 0 and for every $\varphi \in C[0, T]$,

$$
\begin{aligned}
\operatorname{Re}\left\{\int_{[0, T]} \bar{\varphi}(t) \int_{[0, t]} \varphi(t-\tau) d \mu(\tau) d t\right\} & \\
& =1 / 2 \int_{[0, T]} \bar{\varphi}(t) \int_{[t-T, t]} \varphi(t-\tau) d \nu(\tau) d t
\end{aligned}
$$

Proof of Lemma 1.1. Substituting $\mu$ for $\nu$ according to (1.4) one gets

$$
\begin{aligned}
\int_{[0, T]} \bar{\varphi}(t) \int_{[t-T, t]} \varphi(t-\tau) d \nu(\tau) d t & \\
= & \int_{[0, T]} \bar{\varphi}(t) \int_{[0, T-t]} \varphi(t+\tau) d \bar{\mu}(\tau) d t \\
& \quad+\int_{[0, T]} \bar{\varphi}(t) \int_{[0, t]} \varphi(t-\tau) d \mu(\tau) d t .
\end{aligned}
$$


The first term can be written, using Fubini's theorem followed by a change of variables and another application of Fubini's theorem, as

$$
\int_{[0, T]} \bar{\varphi}(t) \cdot \int_{[0, T-t]} \varphi(t+\tau) d \bar{\mu}(\tau) d t=\int_{[0, T]} \varphi(t) \int_{[0, t]} \bar{\varphi}(t-\tau) d \bar{\mu}(\tau) d t .
$$

This combined with (1.7) gives (1.6), and the proof of Lemma 1.1 is complete.

The proof of Theorem 1.1 is now quite straightforward, and we shall only give the following short outline of it. To prove that $\mu \in P D[0, \infty)$ implies $\nu \in P D(-\infty, \infty)$ one first uses the fact that the convolution in (1.3) is invariant under translations of $\varphi$ (i.e. if $\varphi_{s}(t)=\varphi(t-s)$, then $\varphi_{s} * \tilde{\varphi}_{s}=\varphi * \widetilde{\varphi}$ ) to get a function $\varphi_{T}$ with support in $[0,2 T]$, and then one completes the proof using Lemma 1.1 and the density of $\mathcal{D}(0,2 T)$ in $L^{2}(0,2 T)$. For the proof of the converse part one needs the fact that $\nu \in P D(-\infty, \infty)$ implies $\nu=\widetilde{v}$ (this follows from [22, p. 275], and also from Theorem 1.2 below, since by this theorem $\hat{v}$ is real). This means that formula (1.4) expresses $\nu$ in terms of $\mu$, and the positive definiteness of $\mu$ now follows immediately from Lemma 1.1 , because $C[0, T]$ $C L^{2}(-T, T)$.

The class $P D(-\infty, \infty)$ is equivalent to a subclass of Schwartz's positive definite distributions, i.e. the positive definite distributions of order zero. It is clear from (1.3) and $[22$, p. 275] that $P D(-\infty, \infty)$ is contained in the class of positive definite distributions of order zero (replace the function $\varphi$ by its complex conjugate), and using the density of $D(-T, T)$ in $L^{2}(-T, T)$ it is easy to show that every positive definite distribution of order zero is in $\operatorname{PD}(-\infty, \infty)$. Thus the positive definite measures can be characterized in the following way (see [22, pp. $275-276] ; B^{\prime}$ is the class of bounded distributions, in particular $B^{\prime} \subset S^{\prime}$ ):

TheOREM 1.2 (Schwartz). A measure $\nu \in M(-\infty, \infty)$ is positive definite if and only if $\nu \in B^{\prime}$ and $\hat{\nu} \in P(-\infty, \infty)$.

This theorem also remains true if one replaces " $\nu \in B^{\prime}$ " by " $\nu \in S$ ".

Combining Theorems 1.1 and 1.2 one immediately gets (note that by [22, p. 251] and (1.4), $\hat{\nu}=2 \operatorname{Re}\{\hat{\mu}\})$ :

Corollary 1.1. A measure $\mu \in M[0, \infty)$ is positive definite if and only if $\mu \in B^{\prime}$ and $\operatorname{Re}\{\hat{\mu}\} \in P(-\infty, \infty)$.

The mere fact that $\hat{v} \in S^{\prime} \cap P(-\infty, \infty)$ whenever $\nu \in P D(-\infty, \infty)$ imposes a growth condition on $\hat{\nu}$ (see [22, p. 242]). However, exploiting also the fact that $\nu$ is of order zero in a neighborhood of $\{0\}$ one gets a much sharper bound on the size of $\hat{\nu}$ :

Proposition 1.1. Given $\epsilon, \eta>0$ there exists $K(\epsilon, \eta)>0$ such that for every $\nu \in P D(-\infty, \infty)$, 


$$
\sup _{-\infty<\omega<\infty} \hat{\nu}((\omega-\epsilon, \omega+\epsilon)) \leqslant K(\epsilon, \eta)|\nu|((-\eta, \eta)) .
$$

In particular, $\hat{v} \in B^{\prime}$.

This bound is essentially the same as in [2, p. 60].

Proof of Proposition 1.1. Fix $\epsilon, \eta>0$. Pick any $\varphi \in S$ such that $\varphi$ $\geqslant 0, \varphi \geqslant 1$ in $(-\epsilon, \epsilon)$ and $\hat{\varphi} \in D(-\eta, \eta)$ (e.g. take $\psi \in D(-\eta / 2, \eta / 2)$ with $\hat{\psi}(0) \neq 0$, define $\psi_{1}=\psi * \widetilde{\psi}$ (cf. [20, Theorem 7.2(c)] and [22, p. 251]), and then put $\hat{\varphi}(t)=\lambda^{2} \psi_{1}(\lambda t)$ for a sufficiently large number $\lambda[20$, Theorem 7.2(d)]). Define $K=\sup _{t}|\hat{\varphi}(t)|$. Then

$$
\hat{\nu}((-\epsilon, \epsilon)) \leqslant\langle\varphi, \hat{\nu}\rangle=\langle\hat{\varphi}, \nu\rangle\langle K|\nu|((-\eta, \eta)) .
$$

This bound does not change if one translates $\hat{\nu}$, and so (1.8) is true with $K(\epsilon, \eta)$ $=K$. That $\hat{\nu} \in B^{\prime}$ follows immediately from the fact that (1.8) is invariant under translations of $\hat{\nu}[22$, p. 205].

2. Strictly positive definite measures. A certain subclass of the positive definite measures has an interesting property (Theorem 2.1 below), which has some implications for the theory on the asymptotic behavior of the solutions of a nonlinear Volterra equation. Specializing to the case of an integrable function $a$ one has the following lemma:

Lemma 2.1. Let $\varphi \in L^{\infty}(0, \infty)$. Let $a \in L^{1}(0, \infty)$ satisfy $\operatorname{Re}\{\hat{a}(\omega)\}>0$ $(-\infty<\omega<\infty)$. Moreover, suppose that

$$
\sup _{T>0} \operatorname{Re}\left\{\int_{0}^{T} \bar{\varphi}(t) \int_{0}^{t} \varphi(t-\tau) a(\tau) d \tau d t\right\}<\infty .
$$

Define the translates $\varphi_{s}$ of $\varphi$ by

$$
\varphi_{s}(t)= \begin{cases}\varphi(t+s), & t \geqslant-s, \\ 0, & t<-s .\end{cases}
$$

Then $\varphi_{s} \rightarrow 0$ weak $k^{*}$ in $L^{\infty}(-\infty, \infty)$ as $s \rightarrow \infty$. If in addition $\varphi$ is uniformly continuous on $[0, \infty)$, then $\varphi(t) \rightarrow 0$ as $t \rightarrow \infty$.

Rfmark 2.1. The statement about weak ${ }^{*}$ convergence in $L^{\infty}(-\infty, \infty)$ is equivalent to the following: for every $b \in L^{1}(0, \infty)$,

$$
\int_{-\infty}^{\infty} b(-t) \varphi_{s}(t) d t=\int_{0}^{\infty} b(s-t) \varphi(t) d t \rightarrow 0 \quad(s \rightarrow \infty) .
$$

The proof of Lemma 2.1 is essentially the same as the proof of Lemma 1 in [21]; extend $a$ to $(-\infty, \infty)$ as in Theorem 1.1, apply Lemma 1.1, then continue the proof as in $[21, \S 4]$, and finally use two different versions of Wiener's tauberian theorem [20, Theorem 9.7(a)-(b)] . 
REMARK 2.2. It follows from Corollary 1.1 that the measure induced by a function $a \in L^{1}(0, \infty)(d \mu(t)=a(t) d t)$ is positive definite if and only if $\operatorname{Re}\{\hat{a}(\omega)\} \geqslant 0 \quad(-\infty<\omega<\infty)$. Whenever a locally integrable function induces a positive definite measure, we shall henceforth also call this function positive definite.

REMARK 2.3. It is not hard to see that the conclusion of Lemma 2.1 does not necessarily hold if the condition $\operatorname{Re}\{\hat{a}(\omega)\}>0$ is relaxed to $\operatorname{Re}\{\hat{a}(\omega)\} \geqslant 0$ $(-\infty<\omega<\infty)$. The function $a(t) \equiv 0$ provides a trivial example. A less trivial example is the following (cf. [11, Theorem 1]): Take

$$
a(t)=\left\{\begin{array}{ll}
1-t, & 0 \leqslant t \leqslant 1, \\
0, & t>1,
\end{array} \quad \varphi(t)=e^{2 \pi i t}, \quad t \geqslant 0\right.
$$

Then

$$
\operatorname{Re}\{\hat{a}(\omega)\}=\left\{\begin{array}{l}
1 / 2, \quad \omega=0 \\
\left(1 / \omega^{2}\right)(1-\cos \omega), \quad \omega \neq 0
\end{array}\right.
$$

is nonnegative, but not strictly positive everywhere. A short computation yields

$$
\operatorname{Re}\left\{\int_{0}^{T} e^{-2 \pi i t} \int_{0}^{t} e^{2 \pi i(t-\tau)} a(\tau) d \tau d t\right\}=\frac{1}{2 \pi^{2}} \quad(T \geqslant 1)
$$

so (2.1) is satisfied. This shows that the assumption $\operatorname{Re}\{\hat{a}(\omega)\}>0(-\infty<\omega<$ $\infty)$ is indeed essential.

Our present objective is to improve Lemma 2.1 by removing the integrability condition $a \in L^{1}(0, \infty)$, i.e. we would like to replace the function $a$ by a measure $\mu \in M[0, \infty)$. In the view of Remarks $2.2-2.3$ it seems natural to require that $\mu$ be positive definite, and in addition that the derivative of $\operatorname{Re}\{\hat{\mu}\}$ with respect to a Lebesgue measure (i.e. the "density function"), be strictly positive in some sense. There is an almost trivial way of generalizing Lemma 2.1 to a class of "strictly positive definite" measures (Definition 2.1 and Theorem 2.1 below), but it is not immediately clear what this means in terms of Fourier transforms. However, we show below that this leads to a very natural subclass of positive definite measures, i.e. strict positive definiteness of $\mu$ is equivalent to strict positivity of $\operatorname{Re}\{\hat{\mu}\}$ in the appropriate sense. We shall also give a theorem on the strict positive definiteness of nonnegative, nonincreasing and convex functions.

Definition 2.1. A measure $\mu \in M[0, \infty)$ is strictly positive definite $(\mu \in$ $\operatorname{SPD}[0, \infty)$ ) if there exists a function $b \in L^{1}(0, \infty)$ with $\operatorname{Re}\{\hat{b}(\omega)\}>0(-\infty<$ $\omega<\infty)$, such that the measure $\lambda$ defined by $d \lambda(t)=d \mu(t)-b(t) d t(t \geqslant 0)$ is positive definite.

THEOREM 2.1. Let $\varphi \in L^{\infty}(0, \infty)$, and let $\mu \in \operatorname{SPD}[0, \infty)$. Moreover, suppose that 


$$
\sup _{T>0} \operatorname{Re}\left\{\int_{[0, T]} \bar{\varphi}(t) \int_{[0, t]} \varphi(t-\tau) d \mu(\tau) d t\right\}<\infty .
$$

Define the translates $\varphi_{s}$ of $\varphi$ as in Lemma 2.1. Then $\varphi_{s} \rightarrow 0$ weak* in $L^{\infty}(-\infty, \infty)$ as $s \rightarrow \infty$ (see Remark 2.1). If in addition $\varphi$ is uniformly continuous on $[0, \infty)$, then $\varphi(t) \rightarrow 0$ as $t \rightarrow \infty$.

In order to prove Theorem 2.1 it suffices to apply Lemma 2.1 with the function $a$ replaced by the function $b$, given in Definition 2.1.

Definition 2.1 leads to a very short proof of Theorem 2.1, but it leaves one question open: When is it possible to find such a function $b$ ? This problem can be solved in a satisfactory way (Theorem 2.2 below).

DEFInITION 2.2. A measure $\sigma \in M(-\infty, \infty)$ is strictly positive at a point $\omega$ if there exists $\epsilon>0$ such that the measure $\sigma_{\epsilon}$ defined by $d \sigma_{\epsilon}(t)=d \sigma(t)-\epsilon d t$ is positive in $(\omega-\epsilon, \omega+\epsilon)$. A measure $\sigma \in M(-\infty, \infty)$ is strictly positive $(\sigma \in$ $S P(-\infty, \infty))$ if it is strictly positive at every point $\omega \in(-\infty, \infty)$.

REMARK 2.4. Strict positivity of $\sigma$ at the point $\omega$ essentially means that the derivative of $\sigma$ with respect to Lebesgue measure is bounded away from zero in a neighborhood of $\omega$. Clearly $S P(-\infty, \infty) \subset P(-\infty, \infty)$.

THEOREM 2.2. A measure $\mu \in M[0, \infty)$ is strictly positive definite if and only if $\mu \in B^{\prime}$ and $\operatorname{Re}\{\hat{\mu}\} \in S P(-\infty, \infty)$.

The "only if" part follows trivially from Definition 2.1 combined with Corollary 1.1. The nontrivial part of the proof is based on the following lemma, which we state for later use in a slightly more general form than is needed in the proof of Theorem 2.2.

Lemma 2.2. Let $\mu \in P D[0, \infty)$, and let $\Omega \subset(-\infty, \infty)$ be the set where $\operatorname{Re}\{\hat{\mu}\}$ is strictly positive. Then there exists $\varphi \in S$ such that $\hat{\varphi}(\omega)>0(\omega \in$ $\Omega), \hat{\varphi}(\omega)=0(\omega \notin \Omega)$, and the measure $\lambda$ defined by $d \lambda(t)=d \mu(t)-\varphi(t) d t$ $(t \geqslant 0)$ is positive definite.

The proof of Lemma 2.2 is given in $\$ 3$.

The "if" part of Theorem 2.2 now follows immediately. Let $\varphi$ be the function in Lemma 2.2, and define $b(t)=\varphi(t)(t \geqslant 0), b(t)=0(t<0)$. Then $\operatorname{Re}\{\hat{b}\}=1 / 2 \hat{\varphi}(\hat{\varphi}$ is real, and so $\varphi=\widetilde{\varphi}$, which means that $\varphi(t)=\widetilde{b}(t)+b(t)$ $(t \neq 0)$, see [22, p. 251]). Thus $\operatorname{Re}\{\hat{b}(\omega)\}>0(\omega \in \Omega=(-\infty, \infty))$, and $b$ serves as the function required in Definition 2.1.

The following theorem is essentially a special case of Corollary 1.1 and Theorem 2.2:

THEOREM 2.3. Let a be a nonnegative, nonincreasing and convex function on $(0, \infty)$ satisfying $a \in L^{1}(0,1)$. Then $a$ is positive definite. Such a function 
$a$ is strictly positive definite if and only if there exists no $T>0$ such that $a$ is linear in all the intervals $(n T,(n+1) T)(n=0,1,2, \ldots)$.

In view of Corollary 1.1 and Theorem 2.2 one can consider Theorem 2.3 as a reformulation of a known result [5, p. 546] (the real part of the distribution Fourier transform $\hat{a}$ equals the function $\operatorname{Re}\{A(i \omega)\}(\omega \neq 0)$ in [5] plus a positive point mass at $\{0\}$ whenever $a(\infty)>0$ ). For a different version using strong positive definiteness, see [18, Corollary 2.2$]$ and also the counterexample in [18, $\S 4]$, exhibiting a strictly positive definite, nonnegative, nonincreasing and convex function which is not strongly positive definite. Comparing [18, Corollary 2.2] and Theorem 2.3 above it may of course be remarked that the measure $d a^{\prime}(t)$ is purely singular in a very special way (a sum of point masses) whenever $a$ is piecewise linear.

3. Proof of Lemma 2.2. Define $\nu$ as in (1.4). Then $\operatorname{Re}\{\hat{\mu}\}=1 / 2 \hat{\nu}$. Using Theorems 1.1 and 1.2 we can then clearly rewrite Lemma 2.2 in the following form:

LEMMA 2.2a. Let $\nu \in P D(-\infty, \infty)$, and let $\Omega$ be the set where $\hat{v}$ is strictly positive. Then there exists $\psi \in S$ such that $\psi(\omega)>0(\omega \in \Omega), \psi(\omega)=0(\omega$ $\notin \Omega)$, and the measure $\sigma$ defined by $d \sigma(t)=d \hat{\nu}(t)-\psi(t) d t(-\infty<t<\infty)$ is positive.

(As compared to Lemma 2.2, $\psi=\hat{\varphi}$, and $\sigma=2 \operatorname{Re}\{\hat{\lambda}\}$.)

Proof of Lemma 2.2a. We claim that there exist a countable collection of open intervals $I_{j}$ and a sequence of numbers $\epsilon_{j}>0$ such that ( $m$ stands for Lebesgue measure):

(i) $\bigcup_{j=-\infty}^{\infty} I_{j}=\Omega$,

(ii) $\hat{v}-\epsilon_{j} m \geqslant 0$ in $I_{j} \quad(-\infty<j<\infty)$,

(iii) $I_{i} \cap I_{j} \cap I_{k}=\varnothing \quad(i \neq j \neq k \neq i)$.

(iv) each interval has finite length.

It is clear from Definition 2.2 that $\Omega$ is open, and thus $\Omega$ is a countable union of disjoint open intervals. Since a countable union of countable collections of intervals is countable, it suffices to construct the intervals $I_{j}$ in the special case when $\Omega$ is an open interval, say $\Omega=(\alpha, \beta)(-\infty \leqslant \alpha<\beta \leqslant \infty)$. This can be done as follows: Define $\epsilon(\omega)=\sup \{\epsilon \mid \hat{\nu}-\epsilon m \geqslant 0$ in $(\omega-\epsilon, \omega+\epsilon)\}(-\infty<\omega<\infty)$. Then it is easy to show that $\left|\epsilon\left(\omega_{1}\right)-\epsilon\left(\omega_{2}\right)\right| \leqslant\left|\omega_{1}-\omega_{2}\right|$, i.e. $\epsilon$ is continuous. Also $\epsilon(\omega)>0(\omega \in \Omega)$. Take an arbitrary point $\omega_{0} \in(\alpha, \beta)$, and define 


$$
\begin{gathered}
\epsilon_{0}=1 / 2 \epsilon\left(\omega_{0}\right), \alpha_{0}=\omega_{0}-\epsilon_{0}, \beta_{0}=\omega_{0}+\epsilon_{0}, I_{0}=\left(\alpha_{0}, \beta_{0}\right), \\
\epsilon_{1}=1 / 2 \epsilon\left(\beta_{0}\right), \alpha_{1}=\max \left\{\omega_{0}, \beta_{0}-\epsilon_{1}\right\}, \beta_{1}=\beta_{0}+\epsilon_{1}, I_{1}=\left(\alpha_{1}, \beta_{1}\right),
\end{gathered}
$$

and then recursively ("progressing to the right")

$$
\begin{gathered}
\epsilon_{j+1}=1 / 2 \epsilon\left(\beta_{j}\right), \quad \alpha_{j+1}=\max \left\{\beta_{j-1}, \beta_{j}-\epsilon_{j+1}\right\}, \\
\beta_{j+1}=\beta_{j}+\epsilon_{j+1}, \quad I_{j+1}=\left(\alpha_{j+1}, \beta_{j+1}\right) \quad(j \geqslant 1) .
\end{gathered}
$$

We define $I_{j}$ for negative $j$ ("progressing to the left") by

$$
\begin{gathered}
\epsilon_{j-1}=1 / 2 \epsilon\left(\alpha_{j}\right), \quad \beta_{j-1}=\min \left\{\alpha_{j+1}, \alpha_{j}+\epsilon_{j-1}\right\}, \\
\alpha_{j-1}=\alpha_{j}-\epsilon_{j-1}, \quad I_{j-1}=\left(\alpha_{j-1}, \beta_{j-1}\right) \quad(j \leqslant 0) .
\end{gathered}
$$

It follows immediately that (3.1)(ii)-(iv) are satisfied. Also clearly $\bigcup_{j=-\infty}^{\infty} I_{j}$ is an open subinterval of $(\alpha, \beta)$. It then only remains to show that

$$
\lim _{j \rightarrow-\infty} \alpha_{j} \leqslant \alpha, \quad \lim _{j \rightarrow \infty} \beta_{j} \geqslant \beta
$$

So suppose to get a contradiction, that $\lim _{j \rightarrow \infty} \beta_{j}=\gamma<\beta$. This implies that $\lim _{j \rightarrow \infty}\left(\beta_{j+1}-\beta_{j}\right)=1 / 2 \lim _{j \rightarrow \infty} \epsilon\left(\beta_{j}\right)=0$, and thus by continuity, $\epsilon(\gamma)=0$. But this contradicts the fact that $\epsilon(\omega)>0(\omega \in(\alpha, \beta))$. In the same way one proves that $\lim _{j \rightarrow-\infty} \alpha_{j} \leqslant \alpha$.

Once we have the intervals $I_{j}$ satisfying (3.1) the rest of the construction is quite straightforward. Choose functions $\psi_{j} \in S$ such that $\psi_{j}(\omega)=0(\omega \notin$ $\left.I_{j}\right), 0<\psi_{j}(\omega) \leqslant 1 / 2 \epsilon_{j}\left(\omega \in I_{j}\right)(j=0, \pm 1, \ldots)$. Since for every fixed $j$, the function $\delta \psi_{j} \rightarrow 0$ in $S$ as the scalar $\delta \rightarrow 0$ we can pick $\delta_{j}, 0<\delta_{j} \leqslant 1$, such that

$$
d\left(\delta_{j} \psi_{j}, 0\right) \leqslant 2^{-|j|} \quad(j=0, \pm 1, \ldots)
$$

where $d$ stands for an invariant metric in $S$ (see [20, Theorem 7.4(a)]; invariance means that $\left.d\left(\varphi_{1}, \varphi_{2}\right)=d\left(\varphi_{1}-\varphi_{2}, 0\right)\left(\varphi_{1}, \varphi_{2} \in S\right)\right)$. Define $\psi$ by $\psi=$ $\Sigma_{j=-\infty}^{\infty} \delta_{j} \psi_{j}$. This sum converges in $S$ by (3.2), and it follows immediately from the construction that $\psi$ has all the desired properties, in particular, no point of $\Omega$ is covered by more than two intervals, and $\psi(\omega) \leqslant \max \left\{\epsilon_{j}, \epsilon_{k}\right\}$ for $\omega \in I_{j}$ $\cap I_{k}$. This completes the proof of Lemma 2.2a.

4. Laplace transforms of positive definite measures. Since every $\mu \in$ $P D[0, \infty)$ is a tempered distribution (Corollary 1.1 ), we can define the distribution Laplace transform $\mu^{*}$ of $\mu$ (see [23, p. 223]):

$$
\mu^{*}(s)=\left\langle e_{s}, \mu\right\rangle \quad(s \in \Pi=\{s \in \mathrm{C} \mid \operatorname{Re} s>0\}),
$$

where $e_{s}(t)=e^{-s t}(-\infty<t<\infty)$. By [23, Theorem 8.3.2], $\mu^{*}$ is analytic in $\Pi$. 
Theorem 2 in [18] generalizes easily to the present situation:

THEOREM 4.1. Let $\mu \in M[0, \infty) \cap S^{\prime}$. Then the following statements are equivalent:

(i) $\mu \in P D[0, \infty)$,

(ii) $\operatorname{Re}\left\{\mu^{*}(s)\right\} \geqslant 0$ (s $\in$ II),

(iii) $\lim \inf _{s \rightarrow \partial \Pi} \operatorname{Re}\left\{\mu^{*}(s)\right\} \geqslant 0$.

The last statement means explicitly ( $\partial \Pi$ is the boundary of $\Pi$ ):

$$
\begin{aligned}
& \liminf _{s \rightarrow i \omega ; s \in \Pi} \operatorname{Re}\left\{\mu^{*}(s)\right\} \geqslant 0 \quad(-\infty<\omega<\infty), \\
& \liminf _{|s| \rightarrow \infty ; s \in \Pi} \operatorname{Re}\left\{\mu^{*}(s)\right\} \geqslant 0 .
\end{aligned}
$$

The proof of this theorem, like the proofs of Lemma 4.1, Theorem 4.2 and Corollary 4.1 below, will be given in $\$ 5$.

REMARK 4.1. Nohel and Shea [18] write (iii) in a slightly weaker form, taking advantage of an additional smoothness and growth condition on $\mu[18$, line (1.1)] .

Sometimes Laplace transforms are easier to compute than Fourier transforms; in particular it may happen that the Laplace transforms converge in the classical sense for every $s \in \Pi$ (this is assumed throughout in [18]). In this case the criteria (ii) and (iii) in Theorem 4.1 can be quite useful. There also exists a condition similar to Theorem 4.1(iii) which is equivalent to strict positive definiteness. In this connection it is helpful to know that $\operatorname{Re}\left\{\mu^{*}\right\}$ has nontangential limits a.e. on the imaginary axis: Pick any $\alpha>0$, and define

$$
u(\omega)=\lim _{\xi \rightarrow 0+;|\omega-\eta|<\alpha \xi} \operatorname{Re}\left\{\mu^{*}(\xi+i \eta)\right\}
$$

whenever this limit exists. We then have

LEMma 4.1. Let $\mu \in P D[0, \infty)$. Then $u(\omega)$ as defined in (4.1) exists for almost every $\omega \in(-\infty, \infty)$.

REMARK 4.2. The function $u$ in (4.1) is actually a.e. equal to the (measure theoretic) derivative of $\operatorname{Re}\{\hat{\mu}\}$ with respect to Lebesgue measure, i.e. it is a.e. equal to the derivative of the absolutely continuous part of $\operatorname{Re}\{\hat{\mu}\}$. We omit the proof of this fact since we shall not need it explicitly below.

THEOREM 4.2. Let $\mu \in P D[0, \infty)$, and define $u$ as in (4.1). Then the following two conditions are equivalent:

(i) $\mu \in \operatorname{SPD}[0, \infty)$,

(ii) for every $\omega \in(-\infty, \infty)$ there exists $\epsilon>0$ such that $u \geqslant \epsilon$ a.e. in $(\omega-$ $\epsilon, \omega+\epsilon)$. 
Corollary 4.1. Let $\mu \in P D[0, \infty)$. Define

$$
v(\omega)=\liminf _{s \rightarrow i \omega ; s \in \Pi} \operatorname{Re}\left\{\mu^{*}(s)\right\} \quad(-\infty<\omega<\infty)
$$

(i.e. including tangential approach). Then $\mu \in \operatorname{SPD}[0, \infty)$ if and only if $\nu(\omega)>$ $0(-\infty<\omega<\infty)$.

The corresponding result with strict positive definiteness replaced by strong positive definiteness (and the measure $\mu$ absolutely continuous and satisfying the very mild growth condition $[18$, line $(1.1)]$ ) is given in [18, Corollary 2.1$]$.

\section{Proofs of Theorems 4.1-4.2, Lemma 4.1 and Corollary 4.1.}

Proof of Theorem 4.1. The equivalence between (ii) and (iii) follows directly from the maximum modulus principle for harmonic functions. The equivalence between (i) and (ii) can be proved as in [18], but by using Theorems 1.1 and 1.2 together with [2, Theorems 1-2] we can give a shorter proof.

Let $\mu$ be the given measure, and define $\nu$ by(1.4). Take any $\xi>0$, and define $\mu_{\xi} \in M[0, \infty) \cap S^{\prime}, \nu_{\xi} \in M(-\infty, \infty) \cap S^{\prime}$ by $d \mu_{\xi}(t)=e^{-\xi t} d \mu(t)(t \geqslant 0)$, $d \nu_{\xi}(t)=e^{-\xi|t|} d \nu(t)(-\infty<t<\infty)$. Then $\nu_{\xi}=\mu_{\xi}+\tilde{\mu}_{\xi}$. Thus $\hat{\nu}_{\xi}$ is given by the function

$$
\hat{\nu}_{\xi}(\omega)=2 \operatorname{Re}\left\{\hat{\mu}_{\xi}(\omega)\right\}=2 \operatorname{Re}\left\{\mu^{*}(\xi+i \omega)\right\} \quad(\xi>0,-\infty<\omega<\infty)
$$

(see [22, p. 251] and [23, p. 224]).

Now suppose that (i) holds. Theorem 1.1 then tells us that $\nu \in P D(-\infty, \infty)$. Apply [2, Theorem 1] (which by the same proof as in [2] is valid also for positive definite measures) to conclude that $\nu_{\xi} \in P D(-\infty, \infty)(\xi>0)$. Combining this with (5.1) and Theorem 1.2 we find that (ii) is satisfied.

Conversely, suppose that (ii) holds. Theorem 1.2 and (5.1) then imply that $\nu_{\xi} \in P D(-\infty, \infty)(\xi>0)$. Now use [2, Theorem 2] (which also is valid for positive definite measures by the same proof as in [2]) to get $\nu \in P D(-\infty, \infty)$, and Theorem 1.1 then tells us that (ii) is satisfied.

Proof of Lemma 4.1. By Theorem 4.1, $\operatorname{Re}\left\{\mu^{*}\right\}$ is a positive harmonic function on $\Pi$. Map II conformally onto the unit disk with a linear fractional transformation $h$ (e.g. $\left.h(z)=h^{-1}(z)=(1-z)(1+z)^{-1}\right)$. Then the function $g=\operatorname{Re}\left\{\mu^{*}\right\} \circ$ $h^{-1}$ is a positive harmonic function on the unit disk. This means that $g$ can be written as the Poisson integral of a positive measure on the unit circle [19, Theorem 11.19]. Thus $g$ has nontangential limits a.e. on the unit circle [7, p. 34]. Writing $\operatorname{Re}\left\{\mu^{*}\right\}=g \circ h$, and using the fact that $h$ is conformal, we then find that the limit in (4.1) exists for almost every $\omega \in(-\infty, \infty)$, as claimed in Lemma 4.1.

Proof of Theorem 4.2. First suppose that (i) holds. Then by Theorem 4.1, Definition 2.1, and the fact that $b \in L^{1}(0, \infty)$ ( $b$ given by Definition 2.1),

$$
\liminf _{s \rightarrow i \omega ; s \in \Pi} \operatorname{Re}\left\{\mu^{*}(s)\right\} \geqslant \operatorname{Re}\{\hat{b}(\omega)\} \quad(-\infty<\omega<\infty) .
$$


The function $\operatorname{Re}\{\hat{b}\}$ is strictly positive and continuous, and so (ii) is satisfied.

Next we turn to the converse part, and suppose that (ii) holds. Define $\mu_{\xi}$ as in the proof of Theorem $4.1(\xi>0)$. It is an easy, although somewhat tedious, exercise to show that $\mu_{\xi} \rightarrow \mu$ in $S^{\prime}$ as $\xi \rightarrow 0+$. Taking Fourier transforms this means that $\hat{\mu}_{\xi} \rightarrow \hat{\mu}$ in $S^{\prime}$, so in particular $\operatorname{Re}\left\{\hat{\mu}_{\xi}\right\} \rightarrow \operatorname{Re}\{\hat{\mu}\}$ in $S^{\prime}(\xi \rightarrow 0+)$. Choose any nonnegative $\varphi \in D$. Then by Theorem 4.1(ii), (4.1), (5.1) and Fatou's lemma,

$$
\int_{-\infty}^{\infty} \varphi(\omega) u(\omega) d \omega \leqslant \lim _{\xi \rightarrow 0+} \int_{-\infty}^{\infty} \varphi(\omega) \operatorname{Re}\left\{\hat{\mu}_{\xi}(\omega)\right\} d \omega=\langle\varphi, \operatorname{Re}\{\hat{\mu}\}\rangle .
$$

This means that the measure $\lambda$ defined by $d \lambda(t)=d[\operatorname{Re}\{\hat{\mu}\}](t)-u(t) d t(-\infty<t<$ $\infty$ ) is positive, and so by (ii) and Definition $2.2, \operatorname{Re}\{\hat{\mu}\} \in \operatorname{SP}(-\infty, \infty)$. Theorem 2.2 then tells us that (i) is satisfied.

Proof of Corollary 4.1. That $\mu \in \operatorname{SPD}[0, \infty)$ implies $v(\omega)>0(-\infty<\omega$ $<\infty)$ is proved exactly as in the first part of the proof of Theorem 4.2. Conversely, the function $v$ is lower semicontinuous, and so it assumes its minimum on every compact interval. This together with the condition $v(\omega)>0(-\infty<\omega<\infty)$ implies that the function $u$ defined in (4.1) satisfies Theorem 4.2(ii), and thus $\mu \in$ $\operatorname{SPD}[0, \infty)$.

6. Applications to nonlinear Volterra equations. We shall now apply the theory developed in $\S 1-5$ to the nonlinear Volterra integrodifferential equation

$$
x^{\prime}(t)+\int_{[0, t]} g(x(t-\tau)) d \mu(\tau)=f(t) \quad(0 \leqslant t<\infty) ; \quad x(0)=x_{0} .
$$

Here $g$ and $f$ are real-valued functions, $\mu$ is a real, locally finite measure, $x_{0}$ is a real constant, and $x$ is the unknown solution, which is required to be locally absolutely continuous, and satisfy (6.1) a.e. We are basically interested in the asymptotic behavior of $x(t)$ as $t \rightarrow \infty$. Uniqueness of the solution is not assumed.

The following theorem gives the existence and boundedness of solutions of (6.1):

THEOREM 6.1. Suppose that

$$
\begin{gathered}
\mu \in P D[0, \infty), \\
g \in C(-\infty, \infty), \quad f \in L^{1}(0, \infty), \\
|g(\xi)| \leqslant K(1+|G(\xi)|)(-\infty<\xi<\infty), \inf _{-\infty<\xi<\infty} G(\xi)>-\infty,
\end{gathered}
$$

where $G(\xi)=\int_{0}^{\xi} g(\eta) d \eta(-\infty<\xi<\infty)$, and $K$ is some positive constant. Then (6.1) has at least one solution on $[0, \infty)$, and for any solution $x$ the function $g \circ x$ is bounded. If also $\lim \sup _{\xi \rightarrow \pm \infty} G(\xi)=\infty$, then all the solutions of $(6.1)$ are bounded. 
This theorem is proved exactly as [18, Theorem 1(i)], except that by (6.2) the proof of $[18$, line (2.4)] becomes trivial. It contains [18, Theorem 1(i)] as a special case (see [18, Theorem 2], and also Corollary 1.1 and Theorem 4.1 above; it is a trivial exercise to show that it suffices to take real-valued functions $\varphi$ in (1.1) whenever $\mu$ is real). Our smoothness condition on the kernel, i.e. the measure $\mu$, is somewhat weaker, and we have also removed the growth condition on the kernel found in [18, line (1.1)]. For a discussion of earlier results we refer the reader to [18].

Our next theorem concerns the existence of $\lim _{t \rightarrow \infty} g(x(t))$ :

THEOREM 6.2. Let (6.3) hold. In addition, suppose that

$$
\mu \in \operatorname{SPD}[0, \infty)
$$

and that

$$
x \in L^{\infty}(0, \infty)
$$

is a solution of $(6.1)$ on $[0, \infty)$. Define the translates $\varphi_{s}$ of $g \circ x$ by

$$
\varphi_{s}(t)= \begin{cases}g(x(t+s)), & t \geqslant-s, \\ 0, & t<-s .\end{cases}
$$

Then $\varphi_{s} \rightarrow 0$ weak ${ }^{*}$ in $L^{\infty}(-\infty, \infty)$ as $s \rightarrow \infty$ (cf. Remark 2.1). If moreover $x$ is uniformly continuous on $[0, \infty)$, then $\lim _{t \rightarrow \infty} g(x(t))=0$.

To prove Theorem 6.2 it suffices to show that (2.2) holds with $\varphi=g \circ x$, which can be done exactly as in [21], and then to apply Theorem 2.1 (note that uniform continuity of $x$ together with (6.3) and (6.6) implies uniform continuity of $g \circ x)$.

This theorem extends [18, Theorem 1(ii)]. Our assumption on the kernel is weaker, in particular we do not need the growth condition [18, line (1.4)] on $\operatorname{Re}\{\hat{\mu}\}$ (cf. Theorem 4.2 and Remark 4.2 above). Additional conclusions concerning the existence of limits of $x(t)$ and $x^{\prime}(t)$ as $t \rightarrow \infty$ can be obtained as in [18] by restricting the growth of $\mu$ at infinity.

The following theorem gives sufficient conditions, weaker than in [18, Theorem 1(ii)] (see Proposition 6.1 below) for the solution $x$ to be uniformly continuous on $[0, \infty)$ (as in Theorems 6.1-6.2 all functions and measures are real-valued):

THEOREM 6.3. Let (6.3) hold. In addition, suppose that

$$
d \mu(t)=d \lambda(t)+a(t) d t \quad(t \geqslant 0)
$$

where 


$$
\begin{gathered}
\lambda \in P D[0, \infty), \quad|\lambda|([0, \infty))<\infty, \\
a \in C[0, \infty) \cap P D[0, \infty),
\end{gathered}
$$

and let $x$ be a solution of (6.1) satisfying (6.6). Then $x$ is uniformly continuous on $[0, \infty)$.

Proof of Theorem 6.3. Define $\varphi=g \circ x$, and rewrite (6.1) in the form

$$
x^{\prime}(t)=-\int_{[0, t]} \varphi(t-\tau) d \lambda(\tau)-\int_{0}^{t} \varphi(t-\tau) a(\tau) d \tau+f(t) \quad(t \geqslant 0) .
$$

By (6.3) and (6.6), $\sup _{t \geqslant 0}|\varphi(t)|<\infty$, and together with (6.8) this implies that the first term in (6.10) is bounded. That the integral of the last term in (6.10) is uniformly continuous follows from (6.3). Thus it suffices to show that

$$
\sup _{t \geq 0}\left|\int_{0}^{t} \varphi(t-\tau) a(\tau) d \tau\right|<\infty .
$$

To do this, one first argues as in [21] to show that (2.2) holds. Then by (6.7)(6.8),

$$
\sup _{T \geqslant 0} \int_{0}^{T} \varphi(t) \int_{0}^{t} \varphi(t-\tau) a(\tau) d \tau d t<\infty .
$$

Theorem 6.3 is now a consequence of

LemMa 6.1. Let $\varphi$ be locally integrable on $[0, \infty)$, and suppose that (6.9) holds. Then for every $T<\infty$,

$$
\left|\int_{0}^{T} \varphi(T-\tau) a(\tau) d \tau\right|^{2} \leqslant 2 a(0) \int_{0}^{T} \varphi(t) \int_{0}^{t} \varphi(t-\tau) a(\tau) d \tau d t .
$$

We have formulated this lemma for real functions $a$ and $\varphi$. A complex version could also be given.

Proof of Lemma 6.1. Define $b$ as the even extension of $a$ to $(-\infty, \infty)$. Then by (6.9) and Theorem $1.1, b \in C(-\infty, \infty) \cap P D(-\infty, \infty)$. It then follows from Bochner's theorem [1, p. 326] that

$$
b(t)=\frac{1}{2 \pi} \int_{-\infty}^{\infty} e^{i \omega t} d \hat{b}(\omega) \quad(-\infty<t<\infty),
$$

where the measure $\hat{b}$ is positive and finite, i.e. $\hat{b}((-\infty, \infty))<\infty$. Fix $T>0$. Define $\varphi_{T}(t)=\varphi(t)(0 \leqslant t \leqslant T), \varphi_{T}(t)=0(t<0$ or $t>T)$, and let $Q(T)$ denote the integral

$$
Q(T)=\int_{0}^{T} \varphi(t) \int_{0}^{t} \varphi(t-\tau) a(\tau) d \tau d t
$$

Then by Lemma 1.1 and Fubini's theorem,

$$
Q(T)=1 / 2 \int_{0}^{T} \int_{t-T}^{t} \varphi(t) \varphi(t-\tau) b(\tau) d \tau d t=1 / 2 \int_{-\infty}^{\infty} \overline{\left(\varphi_{T} * \widetilde{\varphi}_{T}\right)}(\tau) b(\tau) d \tau .
$$


Thus, using also (6.12), [20, Theorem 7.2(c)], [22, p. 251] and Fubini's theorem,

$$
Q(T)=\frac{1}{4 \pi} \int_{-\infty}^{\infty}\left|\hat{\varphi}_{T}(\omega)\right|^{2} d \hat{b}(\omega)
$$

We now leave this expression for a while, and instead turn to the left-hand side of (6.11). Clearly

$$
\int_{0}^{T} \varphi(T-\tau) a(\tau) d \tau=\left(\varphi_{T} * b\right)(T) .
$$

But $\left(\varphi_{T} * b\right)(T)=(1 / 2 \pi) \int_{-\infty}^{\infty} e^{i \omega T} \hat{\varphi}_{T}(\omega) d \hat{b}(\omega)$ (the proof of this is only based on (6.12), Fubini's theorem and a change of variables), so one finds that

$$
\begin{aligned}
& \left|\int_{0}^{T} \varphi(T-\tau) a(\tau) d \tau\right|^{2}=\frac{1}{4 \pi^{2}}\left|\int_{-\infty}^{\infty} e^{i \omega T} \hat{\varphi}_{T}(\omega) d \hat{b}(\omega)\right|^{2} \\
& \quad \leqslant \frac{1}{4 \pi^{2}}\left[\int_{-\infty}^{\infty}\left|\hat{\varphi}_{T}(\omega)\right| d \hat{b}(\omega)\right]^{2} \leqslant \frac{1}{4 \pi^{2}} \hat{b}((-\infty, \infty)) \int_{-\infty}^{\infty}\left|\hat{\varphi}_{T}(\omega)\right|^{2} d \hat{b}(\omega)
\end{aligned}
$$

where we have also applied Hölder's inequality. Combining this with (6.12)(6.14) we get (6.11), and the proof of Lemma 6.1 is complete.

Although it is not immediately obvious, the conditions (6.7)-(6.9) are weaker than the corresponding conditions in [18, Theorem 1(ii)]:

Proposition 6.1. Let $\mu \in P D[0, \infty)$ be of the form

$$
d \mu(t)=d \sigma(t)+b(t) d t \quad(t \geqslant 0)
$$

where

$$
\begin{aligned}
& |\sigma|([0, \infty))<\infty, \\
& b \in B V[0, \infty) .
\end{aligned}
$$

Then $\mu$ can be written in the form (6.7)-(6.9), with the function a being the restriction to the positive real axis of an entire function.

Proof of Proposition 6.1. Take $\varphi \in S$ such that $\hat{\varphi} \in D(-2,2), \hat{\varphi}(0)=$ 1 , and $0 \leqslant \hat{\varphi}(\omega) \leqslant 1 \quad(-2<\omega<2)$. Define $\nu$ as in (1.4), and then define $a(t)$ $=(\varphi * \nu)(t)(t \geqslant 0)$. By Theorem 1.1, Theorem 1.2, [20, Theorem 7.19(c)] and [20, Theorem 7.22(a)] the function $a$ has all the desired properties, i.e. $a \in$ $C[0, \infty) \cap P D[0, \infty)$, and $a$ is the restriction to the positive real axis of an entire function. Thus it only remains to show that the measure $\nu-\varphi * v$ is finite and positive definite (then restrict this measure to the positive real axis as in Theorem 1.1). However, the positive definiteness of $\nu-\varphi * v$ follows immediately from Theorem 1.2 combined with [20, Theorem 7.19(c)] and the fact that $\hat{\varphi}(\omega) \leqslant 1 \quad(-\infty<\omega<\infty)$, so we only have to prove that the measure $\nu-\varphi * \nu$ is finite. Define $\eta=\sigma+\widetilde{\sigma}$. Then by (6.16) and an application of Fubini's 
theorem the measure $\eta-\varphi * \eta$ is finite, and thus by (6.15) it suffices to show that the function

$$
d(t)=c(t)-\int_{-\infty}^{\infty} \varphi(t-\tau) c(\tau) d \tau \quad(-\infty<t<\infty)
$$

is integrable, where $c$ is the even extension of $b$ to $(-\infty, \infty)$. Using the fact that $\int_{-\infty}^{\infty} \varphi(s) d s=1$ (which is equivalent to $\hat{\varphi}(0)=1$ ) and an integration by parts one can write $d(t)$ in all points of continuity of $c$ as the Lebesgue-Stieltjes integral

$$
d(t)=\int_{-\infty}^{\infty} \psi(t-\tau) d c(\tau),
$$

where $\psi(t)=-\int_{-\infty}^{t} \varphi(s) d s(t<0), \psi(t)=\int_{t}^{\infty} \varphi(s) d s(t \geqslant 0)$. But $\psi \in$ $L^{1}(-\infty, \infty)$, and so $d \in L^{1}(-\infty, \infty)$ (use Fubini's theorem once more). The proof of Proposition 6.1 is complete.

Acknowledgments. The author wishes to thank Professor John Nohel for his encouragement and his suggested improvements in this presentation, and Professor Daniel Shea for his guidance and support in the early stages of this work. This paper constitutes a part of the author's Ph.D. dissertation submitted to the University of Wisconsin, Madison.

\section{REFERENCES}

1. S. Bochner, Lectures on Fourier integrals, Akademische Verlagsgesellschaft, Leipzig, 1932; English transl., Ann. of Math. Studies, no. 42, Princeton Univ. Press., Princeton, N. J., 1959. MR 21 \#5851.

2. J. L. B. Cooper, Positive definite functions of a real variable, Proc. London Math. Soc. (3) 10 (1960), 53-66. MR 22 \#6981.

3. C. Corduneanu, Integral equations and stability of feedback systems, Academic Press, New York, 1973.

4. A. Halanay, On the asymptotic behavior of the solutions of an integro-differential equation, J. Math. Anal. Appl. 10 (1965), 319-324. MR 31 \#579.

5. K. B. Hannsgen, Indirect abelian theorems and a linear Volterra equation, Trans. Amer. Math. Soc. 142 (1969), 539-555. MR 39 \#364.

6. - On a nonlinear Volterra equation, Michigan Math. J. 16 (1969), 365376. MR $40 \# 3225$.

7. K. Hoffman, Banach spaces of analytic functions, Prentice-Hall Ser. in Modern Analysis, Prentice-Hall, Englewood Cliffs, N. J., 1962. MR 24 \#A2844.

8. F. Holland, On the representation of functions as Fourier transforms of unbound. ed measures (to appear).

9. J. J. Levin, The asymptotic behavior of the solution of a Volterra equation, Proc. Amer. Math. Soc. 14 (1963), 534-541. MR 27 \#2824.

10. - On some geometric structures for integrodifferential equations, Advances in Math. (to appear).

11. J. J. Levin and J. A. Nohel, On a nonlinear delay equation, J. Math. Anal. Appl. 8 (1964), 31-44. MR 29 \#445.

12. - Perturbations of a nonlinear Volterra equation, Michigan Math. J. 12 (1965), 431-447. MR 32 \#336.

13. J. J. Levin and D. F. Shea, On the asymptotic behavior of the bounded solutions of some integral equations. I-III, J. Math. Anal. Appl. 37 (1972), 42-82, 288-326, 537575. MR 46 \#5971.

14. S.-O. Londen, The qualitative behavior of the solutions of a nonlinear Volterra 
equation, Michigan Math. J. 18 (1971), 321-330. MR 45 \#2431.

15. On the variation of the solutions of a nonlinear integral equation, $J$. Math. Anal. Appl. (to appear).

16. R. C. MacCamy and J. S. W. Wong, Stability theorems for some functional equations, Trans. Amer. Math. Soc. 164 (1972), 1-37. MR 45 \#2432.

17. R. K. Miller, Nonlinear Volterra integral equations, Benjamin, Menlo Park, Calif., 1971.

18. J. A. Nohel and D. F. Shea, Frequency domain methods for Volterra equations, Advances in Math. (to appear).

19. W. Rudin, Real and complex analysis, 2nd ed., McGraw-Hill Ser. in Higher Math., McGraw-Hill, New York. 1974. MR 49 \#8783.

20. - Functional analysis, McGraw-Hill Ser. in Higher Math., McGraw-Hill, New York, 1973.

21. O. J. Staffans, Nonlinear Volterra integral equations with positive definite kernels, Proc. Amer. Math. Soc. 51 (1975), 103-108.

22. L. Schwartz, Théorie des distributions, new ed., Hermann, Paris, 1966. MR 35 \#730.

23. A. H. Zemanian, Distribution theory and transform analysis. An introduction to generalized functions, with applications, McGraw-Hill, New York, 1965. MR 31 \#1556.

INSTITUTE OF MATHEMATICS, HELSINKI UNIVERSITY OF TECHNOLOGY, SF-02150 ESPOO 15, FINLAND 